\title{
Measurement of Unique Magnetic and Superconducting Phases in Oxygen-Doped High-Temperature Superconductors $\mathrm{La}_{2-x} \mathrm{Sr}_{x} \mathrm{CuO}_{4+y}$
}

\author{
L. Udby, ${ }^{1, *}$ J. Larsen, ${ }^{2}$ N. B. Christensen, ${ }^{2}$ M. Boehm, ${ }^{3}$ Ch. Niedermayer, ${ }^{4}$ H. E. Mohottala, ${ }^{5}$ T. B. S. Jensen, ${ }^{2}$ \\ R. Toft-Petersen, ${ }^{2,6}$ F. C. Chou, ${ }^{7}$ N. H. Andersen, ${ }^{2}$ K. Lefmann, ${ }^{1}$ and B. O. Wells ${ }^{8}$ \\ ${ }^{1}$ Nanoscience Center, Niels Bohr Institute, University of Copenhagen, DK-2100 Copenhagen, Denmark \\ ${ }^{2}$ Department of Physics, Technical University of Denmark, DK-2800 Kongens Lyngby, Denmark \\ ${ }^{3}$ Institut Laue-Langevin, 38042 Grenoble Cedex 9, France \\ ${ }^{4}$ Laboratory for Neutron Scattering, Paul Scherrer Institute, CH-5232 Villigen PSI, Switzerland \\ ${ }^{5}$ University of Hartford, West Hartford, Connecticut 06117, USA \\ ${ }^{6}$ Helmholtz, Zentrum Berlin für Materialien und Energie, D-14109 Berlin, Germany \\ ${ }^{7}$ Center for Condensed Matter Sciences, National Taiwan University, Taipei 10617, Taiwan \\ ${ }^{8}$ Department of Physics, University of Connecticut, Storrs, Connecticut 06269-3046, USA
}

(Received 11 June 2013; published 25 November 2013)

\begin{abstract}
We present a combined magnetic neutron scattering and muon spin rotation study of the nature of the magnetic and superconducting phases in electronically phase separated $\mathrm{La}_{2-x} \mathrm{Sr}_{x} \mathrm{CuO}_{4+y}, x=0.04$, $0.065,0.09$. For all samples, we find long-range modulated magnetic order below $T_{N} \simeq T c=39 \mathrm{~K}$. In sharp contrast to oxygen-stoichiometric $\mathrm{La}_{2-x} \mathrm{Sr}_{x} \mathrm{CuO}_{4}$, we find that the magnetic propagation vector as well as the ordered magnetic moment is independent of $\mathrm{Sr}$ content and consistent with that of the "striped" cuprates. Our study provides direct proof that superoxygenation in $\mathrm{La}_{2-x} \mathrm{Sr}_{x} \mathrm{CuO}_{4+y}$ allows the spin stripe ordered phase to emerge and phase separate from superconducting regions with the hallmarks of optimally doped oxygen-stoichiometric $\mathrm{La}_{2-x} \mathrm{Sr}_{x} \mathrm{CuO}_{4}$.
\end{abstract}

PACS numbers: 74.72.Gh, 74.25.Ha, 74.62.Dh, 76.75.+i

The many active degrees of freedom in transition metal oxides lead to intrinsic complexity with different electronic states being nearly degenerate. As a consequence nanoscale phase separation can be observed in such different materials as the colossal magnetoresistance manganites and high-temperature superconducting (HTSC) cuprates $[1,2]$. A central challenging theme is how dopant disorder influences the details of the phase separation in otherwise electronically similar systems and, e.g., pins fluctuating order [3]. We address this issue by investigating the electronic properties of a HTSC system with two essentially different mechanisms of charge-carrier doping, i.e., mobile oxygen ions and immobile Sr ions.

Starting from the Mott insulating and antiferromagnetic parent compound $\mathrm{La}_{2} \mathrm{CuO}_{4}$ (LCO), replacement of La by $\mathrm{Sr}$ leads to superconductivity above $x=0.055$ in $\mathrm{La}_{2-x} \mathrm{Sr}_{x} \mathrm{CuO}_{4}$ (LSCO) with the highest superconducting transition temperature $T_{c}=38 \mathrm{~K}$ at $x \simeq 0.15$ (optimal doping) [4]. On the other hand, intercalation of a sufficient amount of excess oxygen in $\mathrm{Sr}$-free samples leads to even higher $T_{c} \simeq 42 \mathrm{~K} \mathrm{[5]} \mathrm{and} \mathrm{less} \mathrm{flux} \mathrm{pinning} \mathrm{[6]} \mathrm{in}$ $\mathrm{La}_{2} \mathrm{CuO}_{4+y}(\mathrm{LCO}+\mathrm{O})$. The origin of the differences in superconducting properties lies in the nature of the doping processes: when oxygen-stoichiometric LSCO is formed by cooling through the liquid-solid phase transition at temperatures far above room temperature, a homogeneous but quenched disordered distribution of $\mathrm{Sr}$ on La sites is produced. By contrast, intercalated oxygen remains mobile down to much lower temperatures [7] where it tends to organize in well-ordered superstructures that can be observed in diffraction experiments [8], and over which there is a partial degree of control $[9,10]$. Combining magnetization and muon spin rotation $(\mu \mathrm{SR})$, we have recently discovered that even in samples containing quenched disordered $\mathrm{Sr}$, intercalated oxygen facilitates optimal superconducting properties $\left(T_{c}^{\text {onset }} \simeq 40 \mathrm{~K}\right.$ and weak pinning) [11,12]. It does so by promoting phase separation between regions of the sample that are nonmagnetic (and superconducting) and regions with magnetic order. The local magnetic fields around the muon stopping site are similar [11] to those of the so-called stripe ordered materials (La, Nd) ${ }_{15 / 8} \mathrm{Sr}_{1 / 8} \mathrm{CuO}_{4}$ (LNSCO) and $\mathrm{La}_{15 / 8} \mathrm{Ba}_{1 / 8} \mathrm{CuO}_{4}$ (LBCO) [13]. From elastic neutron scattering (ENS) experiments on these materials it is known that the magnetic order is characterized by two incommensurate magnetic propagation vectors, corresponding to two domains of modulated antiferromagnetic order [14-16]. An ENS study on LSCO $+\mathrm{O}, x=0.09$ revealed similar peaks [17], but a quantitative exposition of the nature and possible evolution of magnetic and superconducting states in $\mathrm{LSCO}+\mathrm{O}$ has been lacking.

In this Letter we present an ENS study of the magnetic properties of $\mathrm{LSCO}+\mathrm{O}$ single crystals covering a broad range of Sr content, and investigate the superconducting properties using high transverse field muon spin rotation (HTF- $\mu \mathrm{SR}$ ). Using neutrons as a bulk-sensitive probe of magnetism, we provide direct evidence for the identity of the magnetic phases of our LSCO + O samples in terms 
of propagation vector and ordered magnetic moment. Moreover, we show that these characteristics are the same as those of stripe ordered LNSCO and LBCO. Further, we find that the superconducting penetration depths of all samples are identical within our experimental errors and of a magnitude similar to that of optimally doped oxygen-stoichiometric LSCO.

The samples studied are the same $x=0.04,0.065,0.09$ single crystals studied in Ref. [11]. They were float-zone grown in an optical furnace and postoxidized (superoxygenated) through wet-chemical methods (see Ref. [8] and the Supplemental Material [18]). The intercalation process was stopped after a long period of oxidation, and always after the sample showed a single $T_{c}^{\text {onset }} \sim 40 \mathrm{~K}$. The ENS studies were performed at the cold triple-axis spectrometers RITA-II and IN14 at the Paul Scherrer Institute, Switzerland and the Institut Laue-Langevin, France, respectively. Both spectrometers employed an elastic scattering mode with $E_{i}=E_{f}=5 \mathrm{meV}$ and 40 minutes of arc horizontal collimation before and after the sample. Be filters removed higher-order contamination scattering from the monochromators. All Miller indices in this work refer to the orthorhombic Bmab notation in reciprocal lattice units (rlu) based on the low temperature lattice parameters (see the Supplemental Material [18]). The muon data were recorded at the General Purpose Surface-Muon Instrument at the Paul Scherrer Institute using a high $(0.3 \mathrm{~T})$ transverse field after fast $(>1 \mathrm{~K} / \mathrm{min})$ cooling, since the superconducting properties are known not to change with cooling rate for the investigated crystals.

To set the stage for LSCO $+\mathrm{O}$, we start by summarizing the magnetic properties of oxygen-stoichiometric LSCO: below $T_{N}$, the magnetic modulation wave vector depends strongly on $\mathrm{Sr}$ content $x$. A quartet of peaks is detected at reciprocal lattice positions $\delta \propto x$ [19] away from the antiferromagnetic point (100). This corresponds to modulated antiferromagnetic (m-AFM) order in the $\mathrm{CuO}$ planes with period $1 / \delta$. For $0.024 \lesssim x \lesssim 0.055$ the spin structure is rotated, i.e., modulated diagonally with respect to the $\mathrm{Cu}-\mathrm{O}$ bonds [19-21]. For $x>0.055$ the modulation is parallel to the $\mathrm{Cu}-\mathrm{O}$ bonds with incommensurability saturating at $\delta \simeq 1 / 8$ for $x \simeq 1 / 8$ [22]. Long-range magnetic order with correlation length $\xi>100 \AA$ is only found for $x \simeq$ $1 / 8$. In striking contrast with these characteristics of LSCO, Fig. 1 shows several key results of our study: in all the investigated superoxygenated $\mathrm{LSCO}+\mathrm{O}$ samples through the Sr doping range $x=0.04-0.09$ at $T \sim 2 \mathrm{~K}$, we have observed a quartet of peaks by ENS at the same positions. The peaks at $\mathbf{Q}=\left(1+\delta_{H}, \delta_{K}, 0\right)$ are compared in Figs. 1(a)-1(c). We find for all $x$ that the peaks are located $\delta_{H} \sim \delta_{K} \sim \delta=0.123 \pm 0.004$ away from the antiferromagnetic point. As the orthorhombic distortion is small (see the Supplemental Material [18]), this corresponds to m-AFM order with a period of 8.1(3) unit cells parallel to the $\mathrm{Cu}-\mathrm{O}$ bonds as is also found in
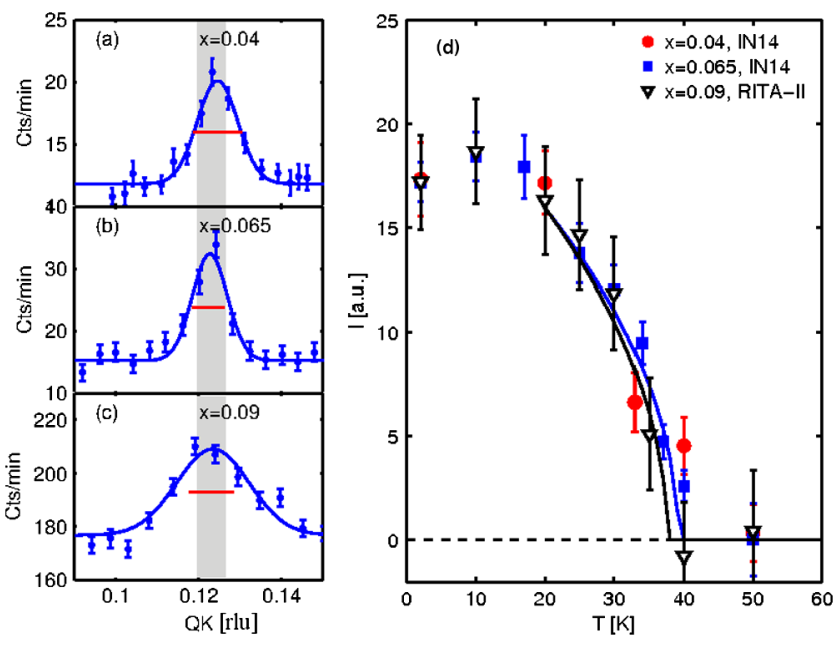

FIG. 1 (color online). (a)-(c) ENS scans in reciprocal space through the m-AFM peak at $\mathbf{Q}=\left(1+\delta_{H}, \delta_{K}, 0\right)$. All data were taken at $T=2 \mathrm{~K}$ at IN14. Solid lines are Gaussian fits and the horizontal red lines show the resolution as found by corresponding scans through structural Bragg peaks. The gray shaded region indicates the average peak position as described in the text. (d) Temperature dependence of the intensity of the m-AFM peaks. Intensities are scaled at $2 \mathrm{~K}$. In order to locate $T_{N}$, the data are fit to a power law with fixed exponent of 0.5 (lines).

oxygen-stoichiometric LSCO, $x \simeq 1 / 8$ [23]. That the incommensurability $\delta$ and the modulation direction is always the same in LSCO $+\mathrm{O}$ regardless of Sr doping $x$ is opposed to what is observed in oxygen-stoichiometric LSCO. The peaks of LSCO + O are sharp and instrumentally resolved for $x<0.09$. For $x=0.09$ there is, however, an $\sim 30 \%$ broadening, resulting from the finite size of the $\mathrm{m}$-AFM domains in the sample [17,24]. These domains are, however, at least $300-400 \AA$ for all $x$. The periodicity and long correlation lengths of the m-AFM signal are similar to those of the zero-field magnetic signal observed in LCO + O $[25,26]$, oxygen-stoichiometric LSCO with $x \simeq 1 / 8$ [23], and the parallel stripes found in LNSCO and LBCO [14-16]. The spin correlation lengths in our $\mathrm{LSCO}+\mathrm{O}$ samples are, however, much larger than in oxygen-stoichiometric LSCO samples with comparable $\mathrm{Sr}$ content [22]. The temperature dependence of the $\mathrm{m}$ AFM peak intensity for $\mathrm{LSCO}+\mathrm{O}$ is shown in Fig. 1(d). It follows the same power-law dependence for all $x$ with transition temperature $T_{N}=39(1) \mathrm{K}$ [27], which is also in contrast to oxygen-stoichiometric LSCO where the intensity does not follow a power-law dependence for the lowest dopings [28] and $T_{N}$ is much smaller and varies in the same Sr doping range [29].

In order to determine the magnitudes of the ordered magnetic moments from the ENS data we need knowledge of the magnetic volume fractions. These can be identified by $\mu \mathrm{SR}$. The muons stop at specific lattice positions and provide a random sampling of the internal field distribution both in the magnetic volume fraction and the vortex state of the superconducting volume fraction. All $\mu \mathrm{SR}$ data 
presented in this Letter were fit to a three-component model for the asymmetry following the procedure outlined in Ref. [30]. Two of the components are temperature dependent and related to the sample. A third component models the background originating from, e.g., muons stopping in the cryostat walls or sample holder, and is assumed to be temperature independent. For details see the Supplemental Material [18]. One temperature dependent component models muon spins that are rapidly depolarized by the presence of ordered moments. The temperature dependence of the corresponding magnetic volume fractions is shown in Figs. 2(a)-2(c). We note that the magnetic volume fractions begin to grow at the same temperatures at which ENS reveals the onset of m-AFM order [see Fig. 1(d)], indicating that truly static magnetic order sets in below $T_{N}$. The second temperature dependent component is slowly relaxing and originates from the nonmagnetic part of the sample. Its temperature dependence is also shown in the top panels of Fig. 2. A slight decrease in precession frequency of this component (see the Supplemental Material [18]) marks the superconducting onset transition at $T_{c}$. At base temperature we assume that all of this component originates from the flux-line lattice in the superconducting volume of the sample. The temperature dependence of the relaxation rate in the nonmagnetic volume is shown in Fig. 2. For all samples we

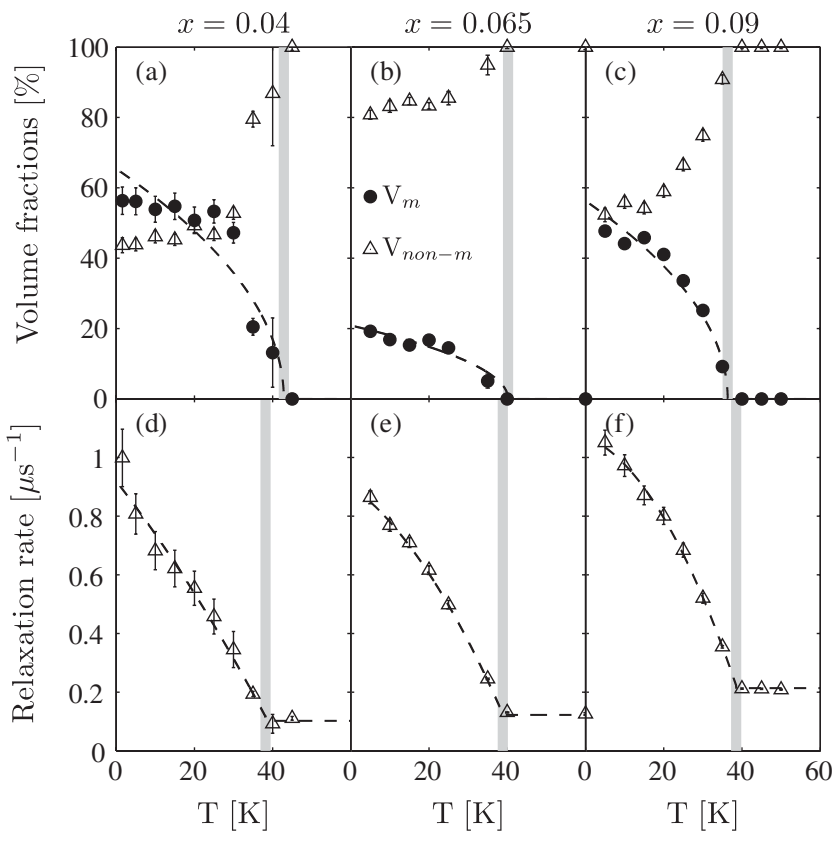

FIG. 2. Results of HTF- $\mu \mathrm{SR}$ experiments. If not visible, the statistical errors are smaller than the data point markers. (a)-(c) Temperature dependence of the magnetic and nonmagnetic volume fractions of the sample. Dashed lines are guides to the eye and gray shaded regions mark $T_{N}$. (d)-(f) Temperature dependence of the spin relaxation rate for muons stopping in the superconducting regions of the sample. Following Ref. [44] the dashed lines are fits to the function $\sigma_{0}\left(1-a T-b T^{2}\right)$ for $T<T_{c}$. Gray shaded regions mark $T_{c}$. observe a similar temperature dependence with relaxation rate $\sigma(T \rightarrow 0) \sim 0.9 \mu \mathrm{s}^{-1}$. This value is the expected relaxation value for a superconducting volume with a penetration depth of at least $\lambda \sim 2500 \AA$ [31] in an optimally doped LSCO sample with a rigid $3 \mathrm{D}$ vortex lattice. It is seen from these data that the relaxation rate increases below $40 \mathrm{~K}$ in all samples, coinciding with the superconducting transition temperature $T_{c}=39(1) \mathrm{K}$. We have confirmed by ac susceptibility measurements that this value of $T_{c}$ is independent of cooling rate. The magnetic and superconducting volume fractions and their transition temperatures are compiled in Table I. We note that $T_{N}$ and $T_{c}$ coincide for all $x$.

We now return to the derivation of the magnetic moment. Table II shows the integrated, mass-normalized peak areas of the m-AFM peaks. The intensity varies substantially, probably due to differences in both magnetic volume fraction and sample mosaic details. Hence, we normalize the m-AFM peaks to the integrated area of a Bragg peak and divide by the magnetic volume fraction to obtain the constant $A$ listed in Table II. Within errors $A$ is the same for all samples, implying they have the same ordered magnetic moment if the same model for the magnetic order can be assumed. We thus turn to the specifics of the symmetryrelated peaks in order to motivate a model for the spin structure. For all LSCO $+\mathrm{O}$ samples we have observed peaks at the same positions $\mathbf{Q}_{m}=\left(1 \pm \delta_{H}, 0 \pm \delta_{K}, 0\right)$ and $\left(0 \pm \delta_{H}, 1 \pm \delta_{K}, 0\right)$ indicating a similar spin structure. For the $x=0.09$ sample, full scans at all of the above mentioned positions were performed and the data fitted to Gaussian line shapes as shown in Fig. 3. The intensities and widths of all the m-AFM peaks are found to be the same within 2 standard deviations as is also observed for the spin stripes in LNSCO [16] and LBCO [32]. We assume that the $\mathrm{m}$-AFM peaks in $\mathrm{LSCO}+\mathrm{O}$ in one direction can be represented by a simple collinear spin stripe model and the other set of peaks in the quartet are generated by an equally favorable $90^{\circ}$ rotated spin stripe orientation. Two m-AFM quartets centered around (100) and (010), respectively, would be observed with similar intensities if either the spin orientation is along the $\mathrm{Cu}-\mathrm{O}$ bonds, as in LNSCO [16] or the spin orientation is along the orthorhombic $b$ axis as in LCO [33] and LCO + O [25] but the crystals are subject to equal size structural twin domains.

For each spin stripe domain we consider an $8 \times 2$ $\mathrm{Cu}$-site unit cell $[\uparrow \downarrow \uparrow \cdot \downarrow \uparrow \downarrow \cdot ; \downarrow \uparrow \cdot \uparrow \downarrow \uparrow \cdot]$ where the moments

TABLE I. Collected $\mu$ SR results for the magnetic and superconducting base temperature volume fractions $V_{m}$ and $V_{\mathrm{SC}}$, respectively, of each sample. $T_{N}$ and $T_{c}$ are determined from ENS (Fig. 1) and $\mu$ SR [Figs. 2(d)-2(f)], respectively.

\begin{tabular}{lcccc}
\hline \hline$x$ & $V_{m}[\%]$ & $V_{\mathrm{SC}}[\%]$ & $T_{N}[\mathrm{~K}]$ & $T_{c}[\mathrm{~K}]$ \\
\hline 0.04 & $56(4)$ & $44(1)$ & $\sim 40$ & $39(1)$ \\
0.065 & $19(1)$ & $81(1)$ & $39(1)$ & $38.7(7)$ \\
0.09 & $47(1)$ & $53(1)$ & $38(2)$ & $37.1(5)$ \\
\hline \hline
\end{tabular}


TABLE II. Mass $(m)$, and mass-normalized m-AFM and nuclear Bragg peak intensities $I_{m}$ and $I_{200}$, respectively. The intensities derive from Gaussian fits to data taken under identical experimental conditions at IN14. The last column shows $I_{m}$ normalized by the product of $I_{200}$ and the magnetic volume fraction $V_{m}$.

\begin{tabular}{lcccc}
\hline \hline$x$ & $m[\mathrm{~g}]$ & $I_{m}[\mathrm{cts} / \mathrm{min} \cdot \mathrm{g}]$ & $I_{200}[\mathrm{cts} / \mathrm{min} \cdot \mathrm{g}]$ & $A=I_{m} / I_{200} \cdot V_{m}$ \\
\hline 0.04 & 0.035 & $2.9(5)$ & $3.7(3) \times 10^{4}$ & $1.4(3) \times 10^{-4}$ \\
0.065 & 0.091 & $2.0(3)$ & $6.4(2) \times 10^{4}$ & $1.6(3) \times 10^{-4}$ \\
0.09 & 0.415 & $1.7(3)$ & $2.0(1) \times 10^{4}$ & $1.8(3) \times 10^{-4}$ \\
\hline \hline
\end{tabular}

are lying in the $\mathrm{CuO}$ plane with angle $\beta$ with $\mathbf{Q}_{m}$. Then the magnetic structure factor is given by $\left|F_{m}\right|^{2}=$ $p^{2} f_{m}^{2} \mu^{2} \sin ^{2} \beta\left|\tilde{F}_{m}\right|^{2}$. For $S=1 / 2$ spins we have $p=$ $0.2696 \times 10^{-14} \mathrm{~m}$, and the form factor for $\mathrm{Cu}^{2+}$ and the geometrical structure factor take the values $f_{m}=0.90(5)$ [34] and $\left|\tilde{F}_{m}\right|^{2}=93.25$ at the m-AFM points (see the Supplemental Material [18]). Based on the experimentally determined factor $A$ in Table II we find the ordered moment in units of Bohr magnetons as

$$
\mu=\sqrt{\left|F_{m}\right|^{2} / p^{2} f_{m}^{2} \sin ^{2} \beta\left|\tilde{F}_{m}\right|^{2}}=0.10(2) \sqrt{C} / \sin \beta .
$$

Assuming that the spins are correlated between neighboring $\mathrm{CuO}$ planes in a manner similar to $\mathrm{LCO}+\mathrm{O}[25]$ the vertical resolution correction gives $C \simeq 1.4$ (see the Supplemental Material [18]) and we obtain $\mu=$ $0.12(2) \mu_{B}$ for spins oriented along [010] $(\sin \beta=0.99)$ as in LCO [33] and $\mu=0.17(3) \mu_{B}$ for spins oriented along [110] $[\sin \beta=0.7(1)]$, as observed in LNSCO [16]. If the spins are assumed to be uncorrelated along the $c$ axis, the scattering intensity is approximately constant along $c^{*}$, and we have $C \simeq 2.7$, and the quoted ordered moments must be corrected by a factor $\sim 1.4$. In the absence of experimental information about the $c$-axis magnetic correlations, we restrain ourselves to the conclusion that the ordered magnetic moments in the magnetic volume fractions of $\mathrm{LSCO}+\mathrm{O}$ are of the same order of magnitude as the those determined for $\mathrm{LCO}+\mathrm{O} \quad\left[\mu=0.15(5) \mu_{B}\right.$ [25] $]$ and stripe ordered $\operatorname{LNSCO}\left[\mu=0.10(3) \mu_{B}[35]\right]$.

In summary, we conclude that superoxygenation facilitates long-range m-AFM order, characterized, within errors, by identical wave vectors, ordered magnetic moments and transition temperatures $T_{N}=39(1) \mathrm{K}$.

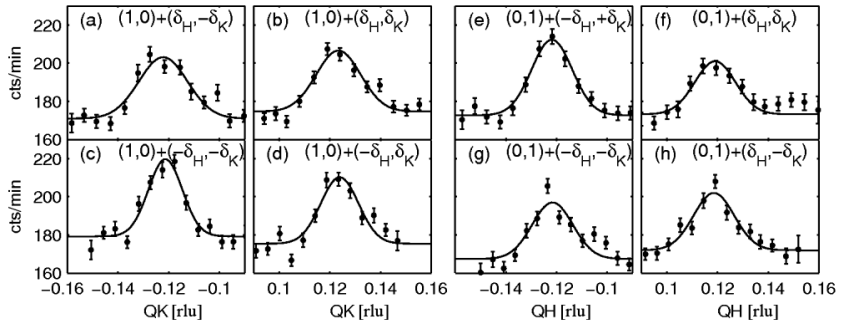

FIG. 3. (a)-(h) Rocking curve scans through the m-AFM peaks of LSCO $+\mathrm{O}, x=0.09$. All lines represent Gaussian fits.
Furthermore, the magnetic transition temperatures in $\mathrm{LSCO}+\mathrm{O}$ are the same within errors whether determined using the local $\mu$ SR probe or bulk-sensitive neutron scattering. All of these properties are in contrast with the behavior of oxygen-stoichiometric LSCO [20,22,29], where $T_{N}$ determined by muons and neutrons differ significantly, reflecting a gradual spin-freezing transition [36]. Our observations are evidence of the existence of a single, long-range ordered $\mathrm{m}$-AFM phase throughout the $\mathrm{Sr}$ doping range $0.04 \leq x \leq 0.09$ in $\mathrm{LSCO}+\mathrm{O}$, the magnetic characteristics of which are similar to those of LBCO $[14,32]$ and LNSCO $[15,16,37,38]$. Since our LSCO + O crystals stay orthorhombic at low temperatures, the stripelike magnetic order is not pinned by the strong ordering field of the low temperature tetragonal phase as in LBCO and LNSCO $[14,15]$. However, the commensurate nature of the ordering still implies a strong coupling to the lattice. We expect that the difference here is that since LSCO + O has a weaker, random disordering field from the Sr dopants it also does not require the stronger lattice ordering field associated with the low temperature tetragonal phase. This picture is consistent with our observation that the LSCO + O sample with the highest Sr content $x=0.09$ has a slightly reduced magnetic correlation length.

Regarding the superconducting phase of $\mathrm{LSCO}+\mathrm{O}$, the transition temperature $T_{c}=39(1) \mathrm{K}$ is the same within errors and is coincident with the magnetic transition temperature for all samples in contrast to $T_{c} \propto x$ in oxygenstoichiometric LSCO [22]. $T_{c}$ is also not suppressed as in the anomalous 1/8 state of LNSCO [39] and LBCO [40] suggesting phase separation rather than competition between the two phases in the same areas of the sample. Furthermore the penetration depth is similar in the superoxygenated system throughout the investigated $\mathrm{Sr}$ range and has a value at least as large as that of optimally Srdoped LSCO. This is in contrast to oxygen-stoichiometric LSCO where the penetration depths for superconducting samples increase with $x$ [41]. The similarity of the temperature dependence of the relaxation rate in $\mathrm{LSCO}+\mathrm{O}$ indicates that the superconducting gap symmetry is similar throughout the $\mathrm{Sr}$ doping range.

These observations prove that a long-range electronic phase separation occurs in LSCO $+\mathrm{O}$ between a $1 / 8$ stripelike magnetic phase and a superconducting phase which is similar to optimally doped LSCO. Thus the phases appear to have different effective charge densities. This interpretation is based on LSCO $+\mathrm{O}$ being in the same family as oxygen-stoichiometric LSCO with respect to the dependence of magnetic ordering, local fields, and superfluid density upon hole concentration. Despite the separated phases in LSCO + O, the magnetic and superconducting regions may still interact, as indicated by the coinciding transition temperatures, in a manner not yet identified. It is possible that the details of this interaction are related to the intriguingly coinciding onsets of 2D superconducting fluctuations and magnetic order in LBCO [42]. In this context, we note that $\mu$ SR and 
magnetization data very similar to ours [11] were recently interpreted in terms of phase separation induced by pressure in LBCO, although in that case $T_{c} \ll T_{N}$ [43].

We thank Brian M. Andersen for helpful discussions. This work was supported by the Danish Agency for Science Technology and Innovation under the Framework Programme on Superconductivity and the Danish Research Council FNU through the instrument center DANSCATT. Work at the University of Connecticut was supported by the U.S. DOE-BES under Contract No. DE-FG02-00ER45801. This work is based on experiments performed partly at the Institut LaueLangevin, Grenoble, France and partly at the Swiss spallation neutron source SINQ, Paul Scherrer Institute, Villigen, Switzerland.

*udby@nbi.dk

[1] E. Dagotto, Science 309, 257 (2005).

[2] G. Alvarez, M. Mayr, A. Moreo, and E. Dagotto, Phys. Rev. B 71, 014514 (2005).

[3] S. A. Kivelson, I. P. Bindloss, E. Fradkin, V. Oganesyan, J. M. Tranquada, A. Kapitulnik, and C. Howald, Rev. Mod. Phys. 75, 1201 (2003).

[4] H. Takagi, T. Ido, S. Ishibashi, M. Uota, S. Uchida, and Y. Tokura, Phys. Rev. B 40, 2254 (1989).

[5] A. Wattiaux, J.-C. Park, J.-C. Grenier, and M. Pouchard, C. R. Acad. Sci. Paris 310, 1047 (1990).

[6] F. Chou, D. Johnston, S.-W. Cheong, and P. Canfield, Physica (Amsterdam) 216C, 66 (1993).

[7] X. Xiong, P. Wochner, S. C. Moss, Y. Cao, K. Koga, and M. Fujita, Phys. Rev. Lett. 76, 2997 (1996).

[8] B. O. Wells, R. J. Birgeneau, F.C. Chou, Y. Endoh, D. C. Johnston, M. A. Kastner, Y. S. Lee, G. Shirane, and J. M. Tranquada, Z. Phys. B 100, 535 (1996).

[9] Y. S. Lee, F. C. Chou, A. Tewary, M. A. Kastner, S. H. Lee, and R. J. Birgeneau, Phys. Rev. B 69, 020502 (2004).

[10] M. Fratini, N. Poccia, A. Ricci, G. Campi, M. Burghammer, G. Aeppli, and A. Bianconi, Nature (London) 466, 841 (2010).

[11] H. E. Mohottala, B. O. Wells, J. I. Budnick, W. A. Hines, C. Niedermayer, L. Udby, C. Bernhard, A. R. Moodenbaugh, and F. C. Chou, Nat. Mater. 5, 377 (2006).

[12] H. E. Mohottala, B. O. Wells, J. I. Budnick, W. A. Hines, C. Niedermayer, and F. C. Chou, Phys. Rev. B 78, 064504 (2008).

[13] B. Nachumi et al., Phys. Rev. B 58, 8760 (1998).

[14] M. Fujita, H. Goka, K. Yamada, J. M. Tranquada, and L. P. Regnault, Phys. Rev. B 70, 104517 (2004).

[15] J. M. Tranquada, B. J. Sternlieb, J.D. Axe, Y. Nakamura, and S. Uchida, Nature (London) 375, 561 (1995).

[16] N. B. Christensen, H. M. Rønnow, J. Mesot, R. A. Ewings, N. Momono, M. Oda, M. Ido, M. Enderle, D.F. McMorrow, and A. T. Boothroyd, Phys. Rev. Lett. 98, 197003 (2007).

[17] L. Udby, N. H. Andersen, F. C. Chou, N. B. Christensen, S. B. Emery, K. Lefmann, J. W. Lynn, H. E. Mohottala, C. Niedermayer, and B. O. Wells, Phys. Rev. B 80, 014505 (2009).
[18] See Supplemental Material at http://link.aps.org/ supplemental/10.1103/PhysRevLett.111.227001 for details of the postoxidation procedure and lattice parameters as well as details in the analysis of the neutron and $\mu$ SR data.

[19] M. Fujita, K. Yamada, H. Hiraka, P. M. Gehring, S. H. Lee, S. Wakimoto, and G. Shirane, Phys. Rev. B 65, 064505 (2002).

[20] S. Wakimoto et al., Phys. Rev. B 61, 3699 (2000).

[21] M. Matsuda, M. Fujita, K. Yamada, R. J. Birgeneau, M. A. Kastner, H. Hiraka, Y. Endoh, S. Wakimoto, and G. Shirane, Phys. Rev. B 62, 9148 (2000).

[22] K. Yamada et al., Phys. Rev. B 57, 6165 (1998).

[23] T. Suzuki, T. Goto, K. Chiba, T. Shinoda, T. Fukase, H. Kimura, K. Yamada, M. Ohashi, and Y. Yamaguchi, Phys. Rev. B 57, R3229 (1998).

[24] L. Udby, P. K. Willendrup, E. Knudsen, C. Niedermayer, U. Filges, N. B. Christensen, E. Farhi, B. O. Wells, and K. Lefmann, Nucl. Instrum. Methods Phys. Res., Sect. A 634, S138 (2011).

[25] Y.S. Lee, R. J. Birgeneau, M. A. Kastner, Y. Endoh, S. Wakimoto, K. Yamada, R. W. Erwin, S. H. Lee, and G. Shirane, Phys. Rev. B 60, 3643 (1999).

[26] B. Khaykovich, Y.S. Lee, R. W. Erwin, S.-H. Lee, S. Wakimoto, K. J. Thomas, M. A. Kastner, and R. J. Birgeneau, Phys. Rev. B 66, 014528 (2002).

[27] We have too few points in the ENS data to fit a proper $T_{N}$ for the small $x=0.04$ crystal, but $T_{N} \sim 40 \mathrm{~K}$ is consistent with the $\mu \mathrm{SR}$ data as discussed later in the text.

[28] S. Wakimoto et al., Phys. Rev. B 60, R769 (1999).

[29] K. Hirota, Physica (Amsterdam) 357-360, 61 (2001).

[30] E. J. Ansaldo, J. H. Brewer, T. M. Riseman, J. E. Schirber, E. L. Venturini, B. Morosin, D. S. Ginley, and B. Sternlieb, Phys. Rev. B 40, 2555 (1989).

[31] Y. J. Uemura et al., Phys. Rev. B 38, 909 (1988).

[32] M. Fujita, Physica (Amsterdam) 481C, 23 (2012).

[33] D. Vaknin, S. K. Sinha, D. E. Moncton, D. C. Johnston, J. M. Newsam, C. R. Safinya, and H. E. King, Phys. Rev. Lett. 58, 2802 (1987).

[34] S. Shamoto, M. Sato, J. M. Tranquada, B. J. Sternlieb, and G. Shirane, Phys. Rev. B 48, 13817 (1993).

[35] J. M. Tranquada, J. D. Axe, N. Ichikawa, Y. Nakamura, S. Uchida, and B. Nachumi, Phys. Rev. B 54, 7489 (1996).

[36] M.-H. Julien, Physica (Amsterdam) 329B-333B, 693 (2003).

[37] J. Chang et al., Phys. Rev. B 78, 104525 (2008).

[38] J. Wen, Z. Xu, G. Xu, J.M. Tranquada, G. Gu, S. Chang, and H. J. Kang, Phys. Rev. B 78, 212506 (2008).

[39] M. K. Crawford, R. L. Harlow, E. M. McCarron, W.E. Farneth, J. D. Axe, H. Chou, and Q. Huang, Phys. Rev. B 44, 7749 (1991).

[40] A. R. Moodenbaugh, Y. Xu, M. Suenaga, T. J. Folkerts, and R. N. Shelton, Phys. Rev. B 38, 4596 (1988).

[41] Y. J. Uemura et al., Phys. Rev. Lett. 62, 2317 (1989).

[42] Q. Li, M. Hücker, G. Gu, A. M. Tsvelik, and J. M. Tranquada, Phys. Rev. Lett. 99, 067001 (2007).

[43] Z. Guguchia, A. Maisuradze, G. Ghambashidze, R. Khasanov, A. Shengelaya, and H. Keller, New J. Phys. 15, 093005 (2013).

[44] J. E. Sonier et al., Phys. Rev. Lett. 72, 744 (1994). 